\title{
Perspective
}

PERSPECTIVE Actualité en histoire de l'art

$4 \mid 2009$

$\mathrm{XX} /\left.\mathrm{XX}\right|^{\mathrm{e}}$ siècles

\section{Les pratiques récentes de mixité entre art actuel et art ancien : le contemporain dans les musées}

The meeting of old and new: bringing contemporary art into traditional museum spaces

Claude Allemand-Cosneau, Claude d'Anthenaise, Thomas Huber, Laurent Salomé et Éric de Chassey

\section{OpenEdition}

Journals

Édition électronique

URL : http://journals.openedition.org/perspective/1258

DOI : $10.4000 /$ perspective. 1258

ISSN : 2269-7721

Éditeur

Institut national d'histoire de l'art

Édition imprimée

Date de publication : 31 décembre 2009

Pagination : 496-509

ISSN : 1777-7852

Référence électronique

Claude Allemand-Cosneau, Claude d'Anthenaise, Thomas Huber, Laurent Salomé et Éric de Chassey, "Les pratiques récentes de mixité entre art actuel et art ancien : le contemporain dans les musées », Perspective [En ligne], 4 | 2009, mis en ligne le 31 juillet 2014, consulté le 10 décembre 2020. URL: http://journals.openedition.org/perspective/1258; DOI : https://doi.org/10.4000/perspective.1258 


\title{
Les pratiques récentes de mixité entre art actuel et art ancien : le contemporain dans les musées
}

\author{
Points de vue de Claude Allemand-Cosneau, Claude D'Anthenaise, Thomas Huber et \\ Laurent Salomé, avec Éric de Chassey
}

Conservateur général du patrimoine, Claude Allemand-Cosneau est directrice du Fonds national d'art contemporain depuis 2001. D'abord conservateur au Musée départemental Dobrée puis au Musée des beaux-arts de Nantes, elle a notamment organisé des expositions sur Paul Delaroche, Gaston Chaissac et Sarkis.

Claude D'Anthenaise, conservateur en chef du Patrimoine, est à la tête du Musée de la Chasse et de la Nature depuis 1998. Commissaire de diverses expositions sur la représentation du paysage ou sur l'image de l'animal, il est l'auteur de plusieurs ouvrages consacrés à ce dernier thème.

Éric de Chassey est professeur d'histoire de l'art contemporain à l'université François Rabelais à Tours, directeur de l'équipe de recherche Intru et directeur de I'Académie de France à Rome depuis 2009.

Artiste qui vit et travaille à Berlin, Thomas Huber est peintre, essayiste et professeur des beaux-arts. II a participé à la conception d'expositions telles que Künstlermuseum, organisée au museum kunst palast à Düsseldorf en 2001.

Après avoir été directeur du Musée des beaux-arts de Rennes, Laurent Salomé est conservateur en chef du patrimoine et directeur des musées de Rouen depuis 2001. II a organisé une centaine d'expositions, privilégiant le XVII ${ }^{\mathrm{e}}$ siècle français, tout en développant une activité croissante en matière d'art contemporain.
Pendant longtemps, les musées ont séparé l'art du passé et l'art du présent, celui-ci étant exposé seulement dans des musées dont le nom même - musée d'art moderne ou d'art contemporain - signalait cette séparation. Depuis quelque temps pourtant, singulièrement en France mais pas uniquement, de nombreux musées se sont mis à montrer au sein de leurs collections permanentes des œuvres d'art créées très récemment voire réalisées spécialement pour l'occasion. Trois conservateurs et un artiste, qui ont tous joué de la mixité au sein d'institutions culturelles, font part de leurs choix personnels et des réactions qu'ils ont pu susciter [Éric de Chassey].

Éric de Chassey. Comment expliquez-vous ce phénomène ? Où et quand trouve$t$-il sa source selon vous et, si vous avez vous-même organisé ce type d'accrochage ou d'installation, quand et où cela s'est-il produit?

Claude D'Anthenaise. Il semble que l'on doive relativiser l'idée d'exclusion de I'art du temps présent dans les mises en scène muséales. Le Musée du Louvre, de manière emblématique, n'a cessé de faire appel à des artistes de renom pour constituer un cadre valorisant les œuvres, depuis l'aménagement, sous le règne de Charles $X$, des salles destinées à recevoir les antiquités égyptiennes, grecques ou romaines jusqu'à la commande, en 1953, d'une peinture à Georges Braque - Les Oiseaux - pour le plafond de I'ancienne antichambre du roi. Il s'agissait alors d'intervenir en marge des collections proprement dites. Mais, en 1947, on expose les œuvres de Picasso dans la Grande Galerie du Louvre, non plus à titre de faire-valoir décoratif des collections anciennes, mais bien pour jouer le jeu des confrontations.

Par la suite et jusqu'à une période récente, les choses se compliquent pour deux raisons apparentes. D'une part, le courant architectural dominant proscrit alors la notion de décor. D'autre part, l'art contemporain, se définissant en rupture par rapport à la tradition, revendique sa "non-intégration ». Les artistes qui font de la subversion ou de l'interrogation sur la nature de l'art le sujet même de leur travail désirent ou s'accommodent de lieux spécifiques qui garantissent leur non-intégration. Des affiches lacérées collectées par les nouveaux réalistes aux extincteurs repeints à l'identique par Bertrand Lavier, les objets extraits du quotidien n'ont pas besoin de la blancheur des cimaises du lieu d'exposition institutionnel pour exister en tant qu'œuvres d'art. 
Mais les choses changent. En ce qui concerne l'évolution actuelle de I'architecture, on assiste à l'inversion de la tendance rationaliste et fonctionnelle : le décor, longtemps cantonné au fameux 1 \% (dispositif instauré en 1951 qui consiste à affecter cette maigre portion du budget de construction d'un édifice public à la commande ou à l'acquisition d'œuvres d'art destinées au bâtiment considéré), revient en force dans l'architecture même des musées (on pense au maniérisme plus ou moins efficace des musées de Bilbao ou du quai Branly). Cette tendance baroque se manifeste dans toute la création artistique. Cédant au goût de la complexité, nous affirmons désormais " $\mathrm{n}^{\prime}$ avoir jamais été modernes ${ }^{1}$. L'œuvre ne se satisfait plus d'une interprétation unique et l'intrusion de l'art contemporain parmi les œuvres du passé pourrait bien correspondre à cet obscur désir de "brouillage ».

Claude Allemand-Cosneau. Élaboré au XVIII ${ }^{e}$ siècle, le système des beaux-arts a fondé le parti pris de présentation des musées chronologiquement et par école. On aurait pu alors imaginer que d'année en année dans un mouvement progressif, l'art moderne puis contemporain ait trouvé sa place sur les cimaises des musées, dans une sorte de continuité fluide. II n'en fut rien puisque l'État, alors principal pourvoyeur des musées en matière d'art vivant, privilégia à partir de 1870 l'art académique et rata toutes les avant-gardes : ni Manet, ni les impressionnistes, ni les Fauves, ni les cubistes, ni les abstraits, ni Duchamp, ni les surréalistes ne furent acquis au moment où ils émergeaient (la première œuvre abstraite achetée par l'État fut une gouache de Kandinsky, Composition IX, acquise en 1937 sous le Front populaire). À Paris, on avait, dès 1818, séparé les vivants des morts avec le Musée du Luxembourg, antichambre du Louvre. La question d'un musée national d'art moderne fut posée dès 1925 , mais sa réalisation prit une vingtaine d'années. Seuls les musées de Grenoble (à partir des années 1920) et le Musée d'art et d'industrie de Saint-Étienne (après la Deuxième Guerre mondiale) comptaient, souvent grâce aux artistes eux-mêmes, une collection moderne qui se développa en collection contemporaine. On comprend donc mieux que des collectivités aient privilégié à partir des années 1960, sous Malraux, la création de musées tout entiers dédiés à l'art contemporain. Pour les musées de beaux-arts existants, il était impossible de combler véritablement par des achats les lacunes historiques des maîtres de l'art moderne ; au mieux une anthologie ou quelques œuvres fortes scandent le parcours, et des sections contemporaines, plus aisées à constituer, sont parfois venues s'adjoindre aux fonds historiques. Dons, legs et dépôts de l'État ont finalement aussi enrichi ces musées en art du Xxe siècle, au point que l'on oublie ces ruptures de l'histoire.

La grande polémique sur l'art contemporain qui débuta dans les années 1990 fut sans doute l'occasion de se poser différemment la question. En effet, les collections permanentes d'art contemporain dans les musées sont présentées à la fin du parcours chronologique des sections beaux-arts, ce qui ne pose pas de problème pour des mouvements déjà historicisés (support-surfaces, les nouveaux réalistes, l'Arte povera, par exemple). En revanche, la proposition de monstration est, pour la production la plus récente, souvent trivialement liée à la disponibilité des œuvres présentes dans la collection et à l'espace même des salles d'expositions, les grandes installations, la vidéo, les photos ou les œuvres graphiques ne permettant pas une présentation pérenne. De même, les rapprochements dans une même salle d'œuvres parfois très diverses sont toujours sous-tendus par un discours, inévitablement incomplet. 
1a. Vue de l'exposition François Morellet au Musée des beaux-arts de Nantes, 1973, hall d'entrée du musée avec 40 lignes au hasard (1970), Aristée tramée (1973), 20 lignes au hasard (1970) et 5 lignes au hasard (1970); b. Aristée tramée : sculpture de Joseph Michel Caillé (1866) et intervention de Morellet (1973) [François Morellet..., 2007, p. 8-9]
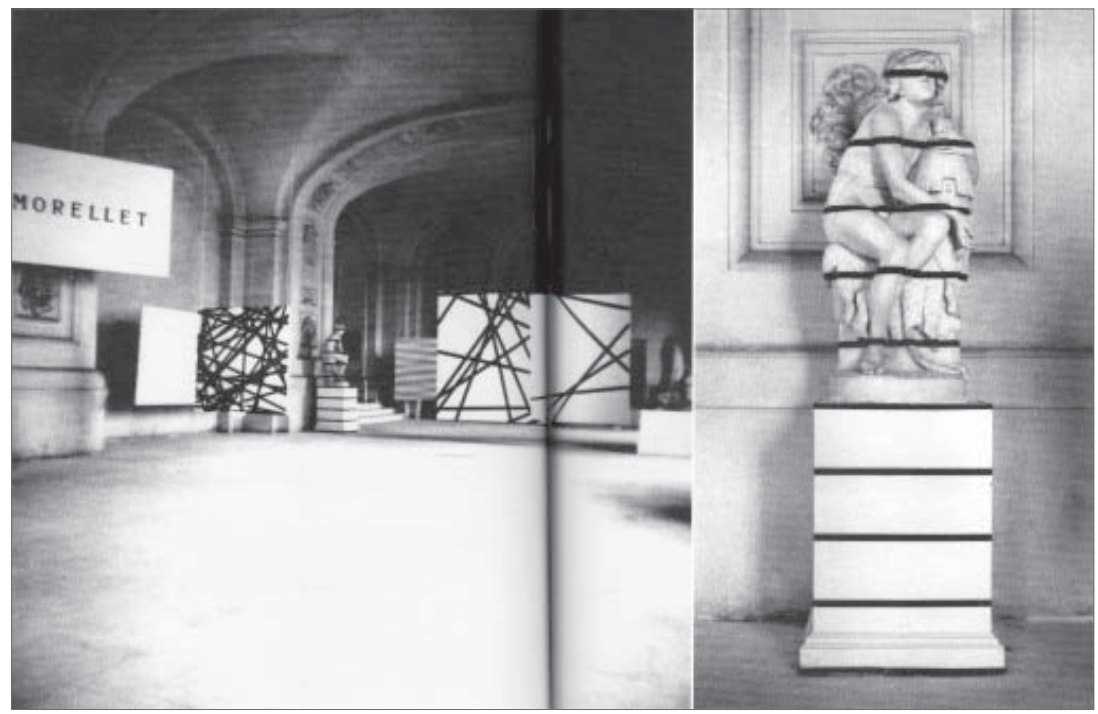

C'est à l'occasion de manifestations temporaires que l'art contemporain s'est le plus facilement insinué dans les salles anciennes, comme à Nantes où, pour la première fois pendant l'été 1985, avec l'exposition Histoires de sculptures, une douzaine d'artistes internationaux investirent plusieurs espaces dans la ville, dont les musées. Paul-Armand Gette installa, en confrère, dans les vitrines du Muséum d'histoire naturelle, ses Perspective Sédimentologique et Perspective de la classification; Buren, avec 3 travaux. De la façade au mur du fond intervint sur les socles des sculptures allégoriques en façade du Musée des beaux-arts et redéfinit la circulation axiale du musée ; dans le hall d'entrée de ce même musée, I'installation monumentale d'Anne et Patrick Poirier intitulée Du regard des statues dialoguait naturellement avec la statuaire du XIXe siècle des collections permanentes. Ces confrontations inédites parurent audacieuses mais justes, sans doute parce que les trois artistes concernés utilisaient un vocabulaire compréhensible à première vue, bien que décalé. Le reste des œuvres contemporaines occupait le patio et les galeries, des espaces traditionnellement dédiés aux collections anciennes décrochées qui avaient été pour l'occasion. Cette exposition fut l'annonce d'une nouvelle ère pour le musée : I'art contemporain devait devenir le moteur de son développement.

Laurent Salomé. II me semble que l'on peut situer le point de départ de la présentation d'œuvres contemporaines dans les collections permanentes dans les années 1970, dans un certain nombre de musées de province qui avaient déjà derrière eux une politique très volontaire en matière d'art contemporain depuis l'après-guerre. Après les abstractions assez sages qui fleurissaient dans les expositions des années 1950 et 1960, des artistes conceptuels ont commencé à proposer des interventions, comme François Morellet à Nantes dès 1973, avec son adhésif noir posé sur les sculptures académiques (Parallèles $0^{\circ}$, Aristée tramé, œuvre éphémère ; fig. 1$)^{2}$. Ce sont moins les musées très orientés vers l'art moderne que les plus traditionnels qui se sont pris au jeu. Sans doute l'effet de surprise rendait-il la chose plus attrayante, et le charme des lieux très typés a tendance à séduire les artistes. II faut aussi citer le rôle des centres d'art installés dans des monuments historiques (comme le Château d'Oiron) et d'autres sites qui, notamment à l'ère Monum, ont familiarisé le public avec le contraste entre la création la plus radicale et le patrimoine ancien. Pour ma part, I'expérience a commencé au Musée des beaux-arts de Rennes en 1995, alors que le Musée de Grenoble où je travaillais précédemment ne s'inscrivait pas dans cette logique. Cela fait une quinzaine d'années que ces « dialogues » me paraissent naturels et que j'en organise en permanence. La collaboration avec les Frac y a beaucoup contribué, avec des confrontations de quelques œuvres ou de collections entières. Le Regard de l'autre à Rouen en 2002, par exemple, confrontait une large sélection de la collection du Frac avec celle du musée. Dans les salles temporaires, I'installation des 

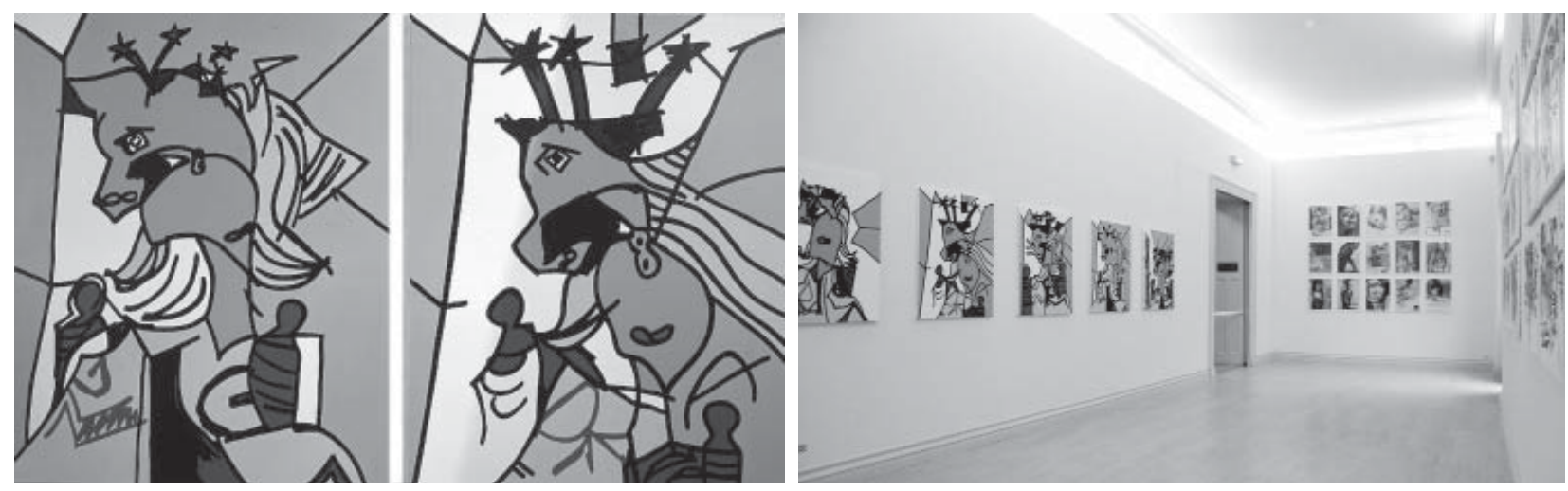

œuvres contemporaines était ponctuée de quelques tableaux anciens descendus de leurs salles (la Belle Zélie d'Ingres dans une section sur la beauté, avec une vidéo de Franck David), le parcours permanent étant en contrepartie truffé d'œuvres du Frac en fonction d'affinités thématiques ou formelles. Ainsi Exit d'Ana Maria Tavarès, une passerelle d'avion servant d'observatoire, était installé dans la "salle du Jubé » entre d'immenses tableaux d'histoire. Aux musées de Rouen, nous accrochons volontiers les dernières acquisitions contemporaines au milieu de l'art ancien, et certaines artistes (Felice Varini, Elmar Trenkwalder) jouent aussi avec l'architecture même du bâtiment. Le regard porté sur l'histoire de l'art, la citation et le deuxième degré constituent l'un des axes de notre programmation en art contemporain. Entre autres, I'artiste-historien de I'art Jean-Philippe Lemée exposait en 2004, sous le titre Roy, Pablo et les autres, des tableaux «faits main ", à savoir des agrandissements par un peintre en lettres de dessins d'amateurs particulièrement maladroits qui copiaient des œuvres de Pablo Picasso et de Roy Lichtenstein (fig. 2).

Thomas Huber. Au tournant du siècle, la ville de Düsseldorf a réorganisé ses lieux d'expositions. Jean Hubert Martin, nommé directeur général du nouveau museum kunst palast, a eu pour tâche d'y mettre en scène des expositions dites populaires et de rappeler à la mémoire du public de Düsseldorf la collection municipale de l'ancien musée d'art. Il a fait appel à Bogomir Ecker, sculpteur originaire de Düsseldorf, et à moi-même pour réorganiser cette collection (fig. 3), un choix qu'il explique à la lumière du contexte historique dans son texte "Musée des charmes " ${ }^{3}$.

Avant même d'être montrée, notre intervention a très vite suscité des controverses et des débats publics dans la ville et jusqu'au Stadtparlement de Düsseldorf, débats qui ont rapidement pris une ampleur nationale puis, à la suite de l'inauguration du nouvel accrochage, internationale. Au centre de la polémique se trouvait la question de savoir si des artistes pouvaient réorganiser les musées, une tâche qui revenait jusqu'alors au personnel scientifique et, à l'arrière-plan, I'indignation que suscita notre présentation, qui bouleversait la chronologie et supprimait la séparation entre les différents départements du musée. Nous avions en effet mêlé et juxtaposé peinture, arts décoratifs et arts noneuropéens du XVI $I^{\mathrm{e}}$ au XXI ${ }^{\mathrm{e}}$ siècle.

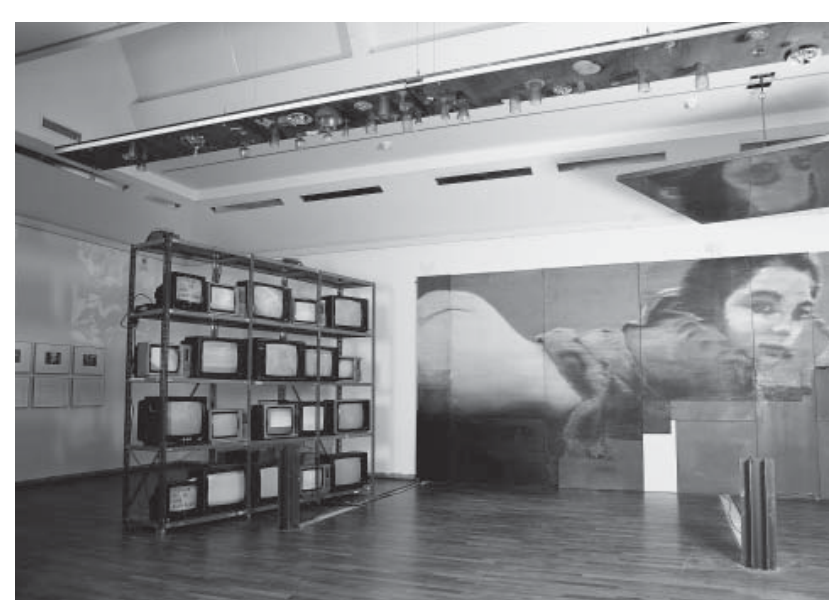

3. Vue du Künstlermuseum, accrochage réalisé par Thomas Huber et Bogomir Ecker, Düsseldorf, museum kunst palast, 2002. et les autres (2004, Collection Frac Bretagne), vue générale et détail, présentée dans l'exposition L'art est sur le côté, Rouen, Musée des beaux-arts, 2004. 
Nous avons dû imposer notre conception malgré une très forte résistance de l'ensemble de la hiérarchie du musée, du conservateur en chef au magasinier, et à l'encontre de présomptions scientifiques et de rituels quotidiens exercés depuis très longtemps. Aujourd'hui encore, je ne peux toujours pas dire ce qui m'a pesé le plus : les combats de tranchées au sein de l'institution ou la polémique publique selon laquelle nous aurions désavoué l'intégrité scientifique du musée.

À mes yeux, les historiens de l'art, en l'occurrence les conservateurs, se considèrent comme des gestionnaires d'un patrimoine collectif et d'un mode de présentation qui répond à des valeurs figées et immuables ; pour nous, les artistes, l'art ancien représente plutôt une source d'inspiration pour une réévaluation continuelle.

Éric de Chassey. Montrer de l'art contemporain au sein de collections anciennes doit-il être interprété comme le signe d'une vision unifiée de la création artistique, qui privilégierait les continuités plutôt que les ruptures, laissant ainsi de côté l'histoire?

Claude Allemand-Cosneau. Montrer de l'art contemporain dans les collections anciennes des musées pose d'emblée la question du statut de l'institution. Traditionnellement le musée est le lieu de conservation, d'étude et de monstration d'un patrimoine préservé, fragmentaire, qui, sorti de son contexte initial, retrouve une histoire dans l'accrochage des œuvres sur les cimaises des salles d'exposition permanentes. L'exercice tendrait-il à s'épuiser ? De nombreux efforts ont été faits pour mieux accueillir le public et pour rendre lisible un discours historique consensuel. Mais la question de la chronologie est là, fixée aussi par la nécessaire organisation matérielle des innombrables collections. Seuls les cabinets de curiosités ou les expositions s'y apparentant autorisent la présentation d'objets d'époques et de natures différentes qui, tout autant sortis de leur contexte que ceux des musées, produisent par leurs rapprochements inattendus un sens nouveau, expression de la vision personnelle du collectionneur ou du commissaire comme, par exemple, les expositions récemment organisées au Palazzo Fortuny pendant les dernières Biennales de Venise. Ouvrir les musées de beaux-arts à l'art contemporain, c'est sans doute afficher qu'une œuvre d'art est une œuvre d'art quel que soit le moment de sa création ; rien n'évoque la période concernée, rien n'explique le contexte de production, comme si l'œuvre contenait en elle-même la justification de son existence. Le musée y gagne évidemment en notoriété car le public et les personnels de l'institution réagissent ; l'événement est commenté. Mais I'histoire n'est pas laissée de côté car ces invitations sont temporaires et le circuit chronologique reprend vite ses droits.

Thomas Huber. Les œuvres d'art dans un musée - une peinture, quel que soit son âge, une sculpture, un objet manufacturé - sont simplement là. Une gravure du $X V I^{e}$ siècle est aussi présente qu'un collage du XXe siècle; en tant qu'objets façonnés, ils ne se distinguent pas dans leur présence matérielle. Dans un musée, il y a aussi des centaines, sinon des milliers d'œuvres qui dorment dans les réserves. Pour l'installation de Düsseldorf, Bogomir Ecker et moi-même avons fouillé les fonds pendant près de deux ans. Nous avons aussi bien vu de l'art ancien que de l'art récent, des objets utilitaires, comme des œuvres d'art qui font autorité. Nous avons sélectionné des pièces en ne tenant compte ni de leurs origines historiques, ni de leurs rapports spécifiques aux genres, mais sous I'angle 
contemporain, par «solidarité artistique». Ce qui nous a guidés, c'est la volonté créatrice de l'artiste et la souveraineté avec laquelle cette volonté s'est exprimée. Notre sélection ne s'est toutefois pas fondée sur des choix subjectifs ou sentimentaux; elle a visé un intérêt pragmatique, celui de comprendre et de faire comprendre la création d'un artefact. Nous sommes en effet tous deux des artistes, et nous avons donc acquis des connaissances que nous avons affinées dans un effort continuel. Notre enseignement dans des écoles de beaux-arts nous a confrontés, en outre, à la nécessité permanente de définir des critères pour expliquer comment une œuvre d'art est faite. Vu sous cet angle, il n'est pas très important de savoir si une œuvre d'art a été réalisée il y a cinq cents ans ou seulement hier.

Laurent Salomé. Il y a bien une continuité dans l'art et même beaucoup moins de renouvellement qu'il n'y paraît ; on peint toujours des vanités, des portraits, des paysages, des supports de méditation, des fantasmes sexuels, etc. Mais le fait d'insérer une œuvre contemporaine dans un milieu décalé est plutôt une façon de dire que I'art d'aujourd'hui est radicalement différent de ce qui l'a précédé. On ne le traite pas de la même manière. En l'isolant et en le rapprochant - parfois de façon incongrue - d'œuvres du passé, on prend des libertés à son égard, on lui donne un statut d'expérience plutôt que de chef-d'œuvre. Je considère aussi ce traitement spécial comme une façon de prendre acte de notre manque de recul. On sous-entend bien sûr que l'œuvre est vouée à occuper une autre place dans I'avenir. Je reste toujours perplexe devant ce besoin que ressentent les institutions spécialisées de montrer le plus vite possible, face à l'art actuel, une froideur distanciée et savante. On ne peut pas mettre en perspective en temps réel, c'est une illusion totale. Si I'approche par confrontations ponctuelles est plus modeste, elle ne convient évidemment pas à tout. II faut que les rapprochements soient plus forts que le décalage. Pour prendre un cas simple, le Musée des beaux-arts de Rouen expose Caterpillar (2002), une sculpture de Wim Delvoye récemment acquise, sorte de pelleteuse gothique hérissée de pinacles et recouverte $d^{\prime}$ un adhésif " décor », dans une salle consacrée au Moyen Âge, à proximité d'une rarissime maquette d'église (Saint-Maclou) du début du XVI ${ }^{\mathrm{e}}$ siècle. Dans ce cas, I'ambiguïté et le clin d'œil sont déjà contenus dans l'œuvre, et la mise en place ne fait qu'enfoncer le clou. Ce qui est intéressant, c'est qu'un large pan de la création actuelle revient sur la question de la mort ou de l'épuisement de l'art, avec cette nécessité de la citation, du retour en arrière, voire du ressassement assumé. C'est un thème qui peut justifier les projets de confrontation, ce pourquoi les artistes-historiens de l'art nous intéressent beaucoup à Rouen. Le grand maître en la matière, qui vient de nous quitter, fut André Raffray avec ses "peintures recommencées ". Nous lui avons consacré, avec le musée de Saint-Brieuc et le Frac Bretagne, une grande rétrospective en 20054. II travaillait encore dernièrement à un projet de diptyque sur la Cathédrale de Rouen de Monet que nous aurions bien sûr accroché à proximité de l'original.

Claude D'Anthenaise. Aujourd'hui, Marcel Duchamp serait largement centenaire. Ses arrières petits enfants ont été élevés dans une tradition qui en vaut bien une autre, celle de la rupture. L'art contemporain, devenu art officiel, s'est embourgeoisé malgré lui. La révolution Duchampienne est considérée scolairement, au même titre que celle des impressionnistes ou celle de la Renaissance. Elle bénéficie du même appareil didactique et fait l'objet des mêmes exégèses. En forçant à peine le trait, on peut dire que la subversion de l'art par les artistes est devenue un exercice 


\section{$X{ }^{e}-X X I^{e}$ SIÈCLES}

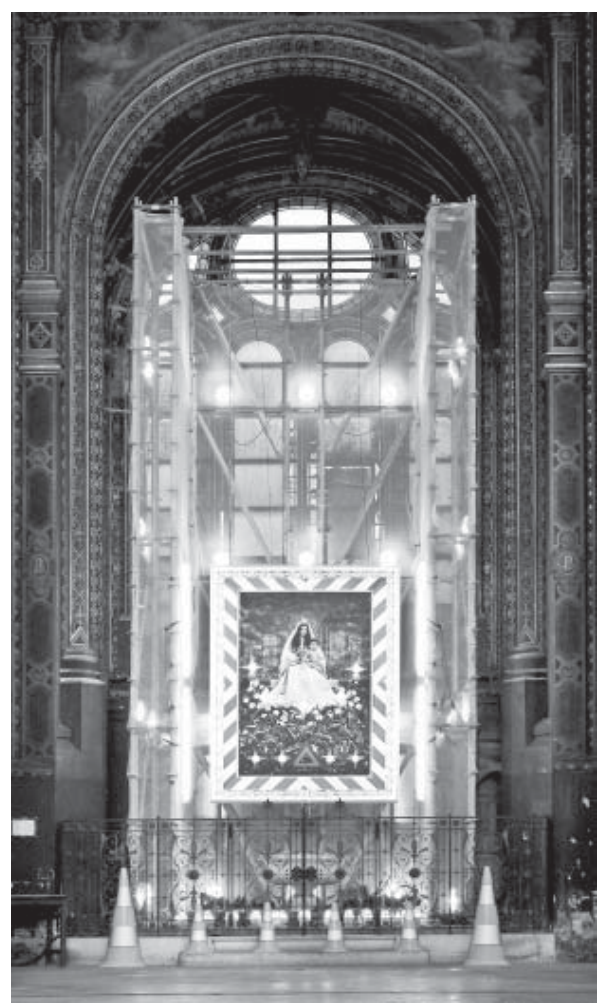

académique. Aussi la notion même de rupture artistique ne suscite plus la même charge émotionnelle auprès du public.

À l'inverse, la sensibilité ambiante pourrait bien réhabiliter la tradition si l'on en juge par le succès de récentes expositions, où tant les hypothétiques ancêtres de Picasso que la descendance légitime et illégitime d'Ingres font courir les foules ${ }^{5}$. La recherche, à travers tous les "salons des refusés ", de l'unité profonde et secrète de la production artistique, motiverait désormais les historiens de l'art et les critiques. À défaut de proximité formelle, on peut alléguer une identité de démarche pour rapprocher des œuvres historiquement incompatibles : la connivence présumée entre l'art de propagande baroque et le marketing propre à Jeff Koons justifie l'insertion de ses œuvres à Versailles. Elles $n^{\prime} y$ viendraient pas pour subvertir un sanctuaire historique mais plutôt pour mettre sa nature en évidence. Dans ce contexte, elles auraient une sorte de vertu pédagogique. Ce peut être une motivation de l'intrusion de l'art contemporain dans les collections des musées. Mais ce n'est pas nécessairement la plus intéressante, ni la plus stimulante pour les artistes.

La révolution Duchampienne reposait en partie sur la "décontextualisation » des objets usuels, promus au statut d'œuvre d'art. De la même manière, l'intégration au musée garantit ce statut en retirant à l'objet toute possibilité d'usage, ne retenant qu'une fonction esthétique ou archéologique. Or l'art contemporain,

4. Pierre et Gilles,

La Vierge à I'Enfant, Paris, église Saint-Eustache, installation in situ réalisée dans le cadre de l'exposition La Force de l'Art 02, 2009. toujours en quête de transgression, s'en prend désormais à lui-même, qu'il s'agisse de ramener la fontaine de Duchamp à sa fonction d'urinoir, ou d'inciter les fidèles à se recueillir devant une Madone de Pierre et Gilles, comme ce fut le cas à SaintEustache dans le cadre de La Force de l'Art 02 (2009; fig. 4). À son tour, le musée peut-il devenir un lieu propice à la subversion en tentant de redonner une valeur d'usage, qu'elle soit décorative, par exemple, illustrative ou pédagogique, aux œuvres contemporaines?

Éric de Chassey. Qui légitime et opère ces inclusions du contemporain dans l'ancien? Des artistes? Des conservateurs ? Y voyez-vous une tension ou une possible contradiction entre un geste artistique qui libérerait les cuvres de certaines contraintes et une muséification qui les ferait entrer, immédiatement ou presque, dans une forme de tradition plus ou moins officielle?

Laurent Salomé. Justement ce paradoxe entre dérision et sacralisation est passionnant. II ne s'est pas affadi depuis Duchamp et le musée est aussi un formidable terrain de recherche pour les artistes. Ce ne sont pas seulement les collections mais aussi I'architecture et I'atmosphère qui permettent des expériences impossibles ailleurs. Le travail sur la muséographie elle-même est particulièrement riche depuis une dizaine d'années et les propositions des artistes sont aussi l'occasion de repenser entièrement le "donner à voir ». Lors de l'exposition À travers le miroir organisée au musée de Rouen en $2000^{6}$ (vous ne m'en voudrez pas de prendre tous mes exemples dans la maison, l'idée étant d'apporter un témoignage), Daniel Buren s'empara des sculptures du XIX siècle, déjà savamment mises en scène dans le « Jardin des sculptures ", pour les inclure dans des modules combinant panneaux rayés et faces éfléchissantes, grâce auxquels on pouvait apercevoir la sculpture vue de haut (fig. 5). 
Ceux-ci furent qualifiés de cabines de plage par les visiteurs les plus réfractaires, dont les commentaires furent d'une grande violence. Des artistes s'approprient même le champ de la médiation, de la didactique. Notre exposition récente $d^{\prime}$ Alain Sonneville et de Pierre-Claude De Castro, Leur entrée dans l'art (Rouen, Musée des beaux-arts, 2009-2010), était constituée presque exclusivement de cartels. Quant à la légitimation, comme pour toute forme d'art, elle est produite conjointement par les artistes, les responsables d'institutions et les critiques, mais elle ne représente plus aucun enjeu. Le risque aujourd'hui est

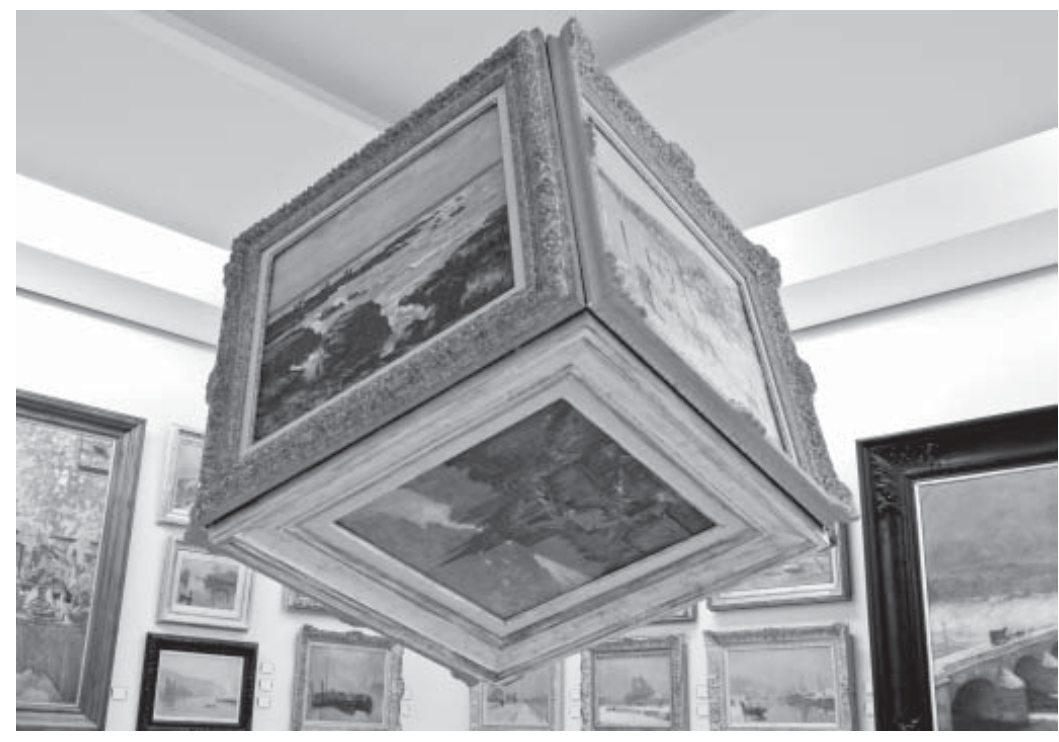
plus celui de la tarte à la crème que celui du scandale : les confrontations entre art contemporain et collections sont devenues hélas une forme banale d'animation culturelle. Les artistes honnêtes s'en méfient et veillent à rester dérangeants. Le duo rouennais Bertran Berrenger, qui a sévi en 2007 à travers tout le musée avec des propositions absolument magistrales, avait même joué sur l'angoisse d'être assimilé à l'École de Rouen. Ce mouvement impressionniste local d'abord d'avant-garde (vers 1880) a perdu sa définition stricte pour s'éterniser loin dans le XXe siècle dans un interminable affadissement de l'impressionnisme, d'où la réputation ambiguë de cette école aujourd'hui. La solution fut de constituer un cube fait d'un assemblage de six véritables tableaux de l'École de Rouen (empruntés à des collections privées) et de le suspendre de biais dans la salle elle-même cubique et tapissée de tableaux de cette école (fig. 6). Le résultat, très spectaculaire, incarnait à la fois une réflexion sur la fermeture d'un milieu, sur le rapport peinture-sculpture, et sur la valeur, le respect et la muséification.

Claude Allemand-Cosneau. L'initiative d'inviter des artistes contemporains est principalement le fait de directeurs des musées, qui délèguent éventuellement cette action à un conservateur en charge de l'art contemporain, comme c'est le cas au Louvre. La demande à l'artiste est généralement précise même si elle est ouverte. L'intervention est très contextualisée puisqu'elle a lieu au sein des collections elles-mêmes, dans lesquelles il s'agit de choisir un domaine ou des œuvres particulières, de réagir, de s'intégrer ou de s'opposer, d'établir des correspondances et de répondre en quelque sorte à un environnement artistique. L'artiste est sans doute le plus à même de maîtriser une telle intrusion et de la légitimer.

Le musée a longtemps été le lieu de formation des artistes, et les plus grands se sont souvent inspirés des maîtres du passé. L'exposition Picasso et les maîtres était à ce titre exemplaire et Ingres et les modernes à Montauban, qui démontrait I'extraordinaire postérité du peintre, plus riche encore ${ }^{7}$. Chaque artiste d'aujourd'hui pourrait-il faire l'objet d'une invitation au Louvre ? Ou bien serait-ce trop évident ? Et si dans un musée, imaginaire bien sûr, ces œuvres contemporaines étaient conservées ensemble avec les originaux d'Ingres, aurait-on idée de les présenter conjointement de manière permanente ? Il y a fort à parier que non car les artistes 
7. Le "Cabinet du sanglier», Paris, Musée de la Chasse et de la Nature : poire à poudre en os, XVII siècle; crâne de sanglier ; coupe grecque, $V^{e}$ siècle avant J.-C., dépôt du Musée du Louvre ; Jean-Luc Chapin, Traces, ensemble de six photographies, 2005. contemporains seraient alors réduits à cette seule référence, ce que le prétexte d'une exposition temporaire autorise. La question de l'instrumentalisation de l'art contemporain par le musée pourrait se poser si cette note contemporaine devenait systématique.

Thomas Huber. À partir du XIX $X^{\mathrm{e}}$ siècle seulement et surtout à partir du XXe siècle, des œuvres ont été créées en vue de leur présentation possible et de leur conservation dans un musée. Les œuvres plus anciennes qui nous sont données à voir aujourd'hui dans ce type d'institution sont, en revanche, arrachées à leur contexte initial, très différent. Un autel gothique dans un musée n'a plus aucune fonction dévotionnelle, mais se voit jugé sur ses qualités artistiques. Même la contextualisation et l'appréciation chronologique scientifique d'un tel objet culturel ne rétablissent pas sa fonction originelle ; elle lui rend hommage dans un contexte nouveau, marqué par les expériences d'aujourd'hui. Si une œuvre d'art dans un musée nous parle, c'est parce que nous la ressentons directement, immédiatement. Or, nous perdrions beaucoup en percevant cette œuvre uniquement comme un document historique, ce qui reviendrait à lui soustraire son efficacité.

Claude D'Anthenaise. Le Musée de la Chasse et de la Nature est un terrain favorable pour ce genre d'expérience : musée de société, consacré à l'illustration du rapport de l'homme à la nature, il n'a pas pour vocation première de questionner sur la nature de l'œuvre d'art. Dès l'origine, il était conçu comme une maison, celle d'un collectionneur amateur. L'accrochage des œuvres voulu par les fondateurs exprimait des partis pris, des choix subjectifs, des rapprochements audacieux, tels que la présentation hétéroclite, sous un plafond peint par Bernard Lorjou en 1967, de statuaire animalière du XIX siècle, d'animaux naturalisés et d'objets ethniques africains. Mais, après une quarantaine d'années d'existence, le musée s'assoupissait sans parvenir à renouveler l'intérêt du public.

Au cours de la période de réflexion préalable à la rénovation de la muséographie, une expérience s'est avérée déterminante. En 1999, Numéro vert de Gloria Friedmann, installation provisoire destinée à la cour du musée, est venu renouveler le regard sur les collections. Aux yeux d'un nouveau public attiré par I'art contemporain, c'était tout le musée, avec l'hétérogénéité de ses collections et le caractère insolite de son accrochage, qui pouvait être perçu comme une installation. L'intérêt porté par ces nouveaux visiteurs a incité à assumer, voire même à

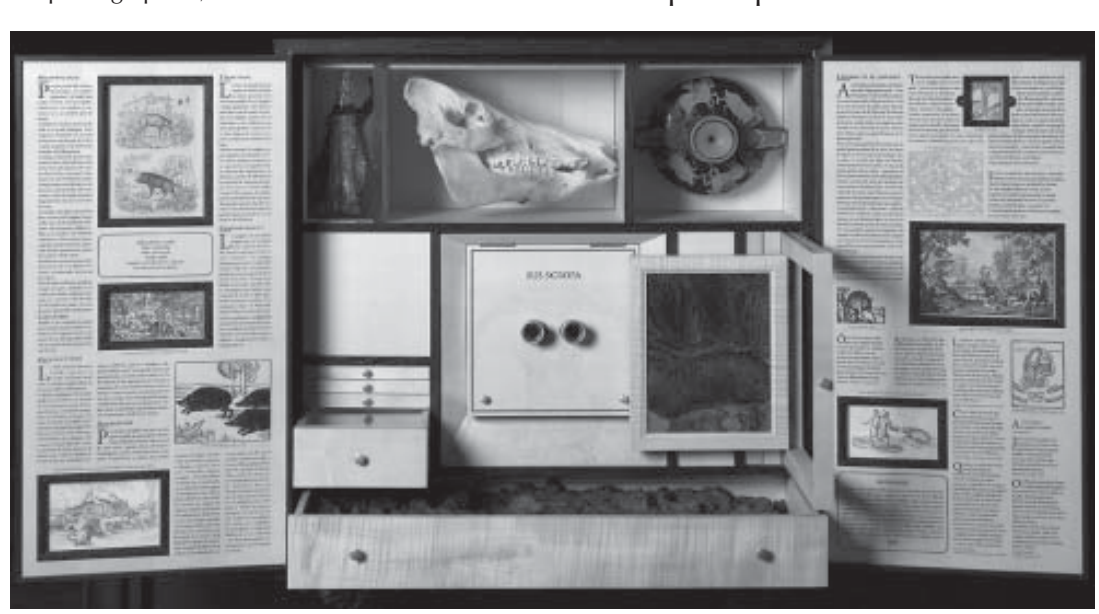

amplifier, les particularités du lieu. La rénovation a été le prétexte à un exercice de mise en scène avec un aspect décoratif très poussé. Cette muséographie n'exclut pas I'humour, qui permet de mettre sur le même plan une coupe antique de la collection Campana (dépôt du Musée du Louvre), un travail photographique contemporain de Jean-Luc Chapin et un excrément de sanglier (fig. 7). Multipliant les pièges et les chausse-trapes - les œuvres qui n'ont pas l'air d'en être 
et celles qui n'en sont pas alors qu'elles en ont l'air - le musée cherche à déstabiliser le visiteur. Au questionnement propre à un certain art contemporain, "qu'estce que l'art », tend à se substituer une nouvelle interrogation : «qu'est-ce qu'un musée? ». Parmi les diverses aberrations naturelles que le visiteur est appelé à découvrir se trouvent quelques spécimens d'étude naturaliste, tels les indices indiscutables de l'existence de la licorne réunis par Joan Foncuberta, Maïder Fortuné, Sophie Lecomte, Jean-Michel Othoniel... Les appareils pédagogique et signalétique sont le terrain privilégié de cette «mise en cause » du musée. Parmi les différentes expériences en ce domaine, on peut noter la

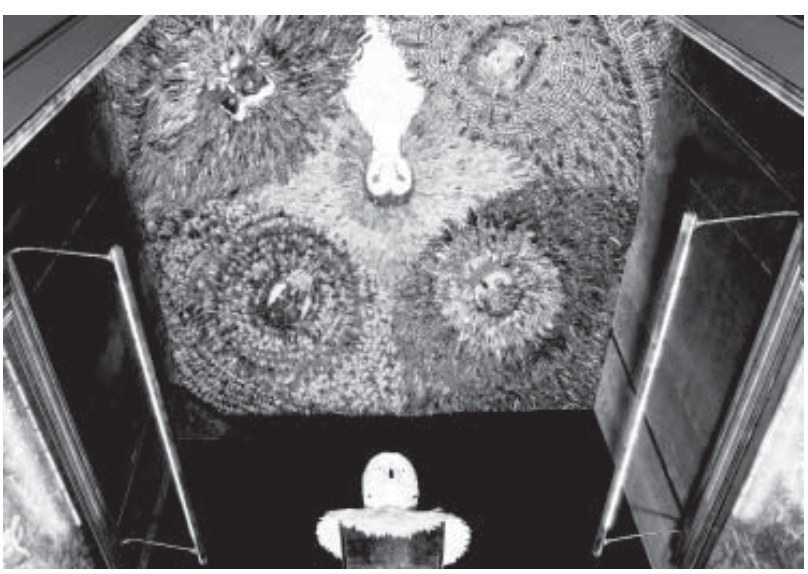

8. Le "Cabinet de Diane", Paris, Musée de la Chasse et de la Nature : Jan Fabre, Le ciel des hiboux, 2007 ; Peter-Paul Rubens et Jan Brueghel, Diane et ses nymphes s'apprêtant à partir pour la chasse et Diane et ses nymphes observées par des satyres, vers 1625 .

la conception muséographique ainsi que dans le choix des œuvres, mais cette liberté s'accompagne de fortes contraintes pour les artistes. Les commandes du musée étant en effet assorties d'un cahier des charges très précis au regard du contexte et du sens de l'œuvre, les artistes sont invités à s'insérer dans un discours structuré et dans un cadre architectural et décoratif élaboré ${ }^{8}$. Certains d'entre eux ont botté en touche ou décliné la commande, notamment à cause de cet enjeu « d'usage » qu'ils n'estimaient pas compatible avec la nature de leur travail. D'autres ont pu trouver un caractère stimulant à ces contraintes " d'intégration », comme Jan Fabre, qui a accepté de réaliser un plafond pour un cabinet dont le thème, la couleur et la matière étaient précisément définis (fig. 8).

Eric de Chassey. Dans quelle mesure l'inclusion d'auvres contemporaines au sein de collections plus anciennes produit-elle du sens? Pensez-vous qu'elle rende contemporain l'ancien, dans une époque que l'on peut interpréter comme marquée par une vogue de l'art contemporain (si l'on en juge par son apparition dans les magazines de mode et dans les prescriptions sociales des parties les plus riches et les plus éduquées de la population) ? Ou pensez-vous qu'elle rende plus acceptable le contemporain, qui conserverait autrement un pouvoir de choc et s'affronterait au refus, par principe, de ce qui est nouveau de la part du public traditionnel des musées? Claude D'Anthenaise. Le jeu auquel se sont prêtés les artistes contemporains a indubitablement permis de développer le public du Musée de la Chasse et de la Nature, pour reprendre cet exemple. Celui-ci s'est ouvert aux amateurs d'art contemporain, qui paraissent sensibles et favorables à ce détournement des codes muséographiques. De manière générale, l'image de l'institution a changé : son caractère artistique s'est affirmé dans l'opinion de visiteurs qui étaient auparavant rebutés par ce qu'ils présumaient de sa vocation technique. Cette ouverture pouvait présenter un risque : I'enjeu de la nouvelle muséographie était de satisfaire le public traditionnel en quête d'informations précises sur les animaux ou sur les pratiques de chasse. Dans cette optique, l'élaboration d'un discours structuré sur les collections et sur leur présentation permet de donner une cohérence 
9. Sarkis, Au commencement le toucher, 2005, Colmar, Musée d'Unterlinden.

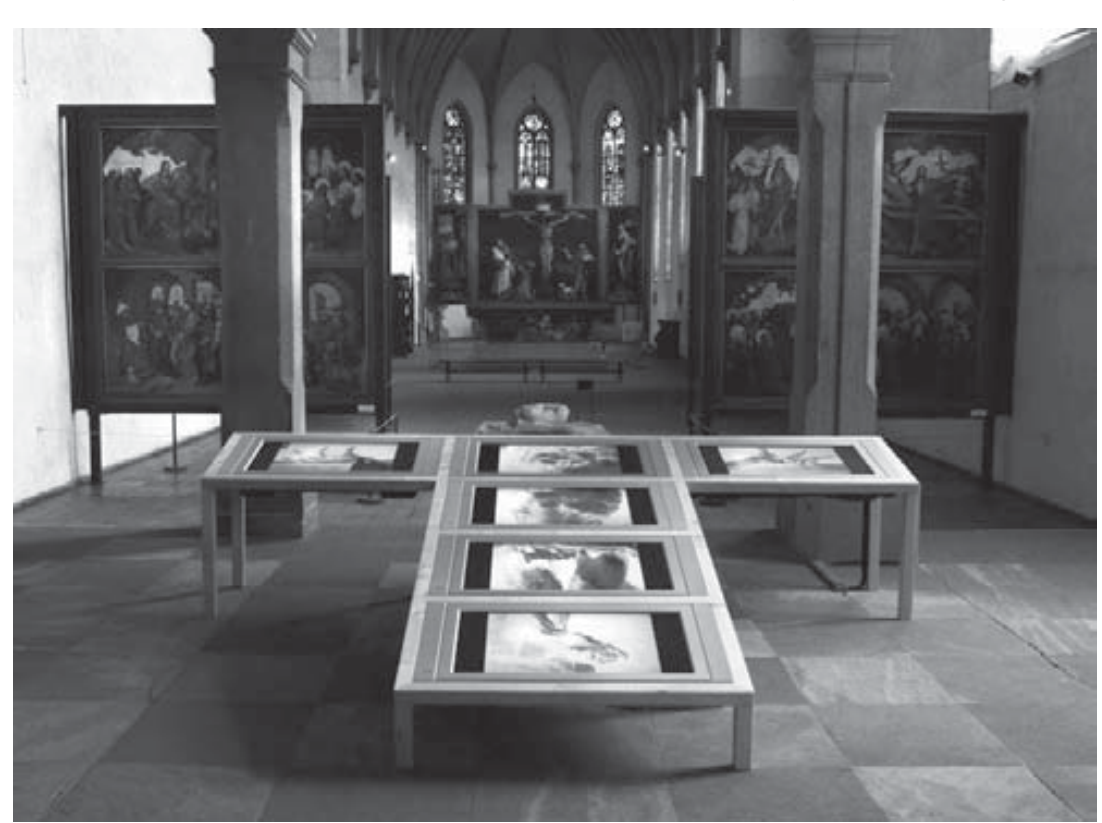

à la visite. Ce discours, transmis par la médiation mise en place, privilégie désormais une interprétation des œuvres : celle qui concerne la relation de l'homme à l'animal envisagée dans une perspective historique. Cet alibi anthropologique contribue jusqu'à un certain point à désamorcer les réactions d'hostilité du public rétif à l'art contemporain. Comment évoquer, en effet, le rapport de l'homme à I'animal à l'époque contemporaine en l'illustrant exclusivement avec des œuvres du passé ? Dans la mesure où ils ont conscience de cette impossibilité, les visiteurs sont moins enclins à dénoncer la " gratuité » des interventions d'artistes de notre temps.

Qu'elles aient une utilité décorative ou une fonction pédagogique, les créations contemporaines distillées parmi les collections du Musée de la Chasse et de la Nature y ont une "valeur d'usage ». Au lieu de jouer le jeu des « intrus », tout contribue à les intégrer. Le traitement signalétique propre au musée contribue à cette homogénéisation en invitant l'observateur à voir avant de savoir. Dans ce lieu voué à la chasse, distinguer l'anachronisme peut être l'objet d'une quête ludique.

Claude Allemand-Cosneau. Lorsqu'un artiste intervient en créant en réponse à une œuvre ancienne, il nous informe sur son art, ses intérêts, sa propre vision des artistes du passé. À ce titre, il génère de nouveaux liens. Notre contemporain est sans arrêt nourri du passé et de l'ensemble de nos connaissances, comme un immense terreau pour la pensée et l'action d'aujourd'hui. Mais la nécessité pédagogique du classement dans nos musées au regard d'une histoire de l'art européenne, toujours approfondie mais finalement stable malgré des redécouvertes, ne nous permettra sans doute pas facilement de rebattre les cartes, sauf peut-être dans une présentation thématique comme l'avait fait le MoMA à New York en 1999-2000, dans ModernStarts: People, Places Things. Traitant de la naissance de la modernité de 1880 à 1920, ces trois accrochages proposaient en outre des rapprochements avec des œuvres contemporaines destinées à démontrer la persistance des thèmes. Tout le siècle était ainsi traversé, un adolescent de Cézanne voisinant avec une photo de Renike Dijkstrat. À l'étage de Things, une immense peinture murale de Martin Craig-Martin, Objects, Ready and Not (1999), créait le décor avec une succession d'objets en trompe-l'œil, parfois redondants avec les œuvres exposées. L'art contemporain est en vogue, certes, mais à chaque époque le temps présent a été valorisé. Présentée à dose homéopathique dans les collections anciennes, le temps d'une exposition temporaire, la création d'aujourd'hui est certainement une tentative pour faire évoluer le musée et en renouveler l'intérêt, mais c'est probablement l'institution qui espère en recueillir le plus grand bénéfice et non pas les œuvres anciennes elles-mêmes. 
Il arrive cependant que ce soient les artistes qui intègrent à leur propre travail des œuvres du passé, y compris à l'intérieur du musée, comme Sarkis par exemple, dont la réflexion trouve toujours un point d'ancrage dans l'histoire. Sa fascination pour le retable d'Issenheim de Grünewald (vers 1512-1515) conservé au musée d'Unterlinden à Colmar l'a conduit à intervenir en 2005 dans l'espace même du musée, devant le retable, permettant ainsi au spectateur de voir les deux œuvres en même temps (fig. 9). Sarkis s'appropriait l'œuvre de Grünewald avec un infini respect, au point de reconstruire dans le même bois un caisson en croix dans lequel s'inscrivait en vidéo sa délicate intervention : I'artiste posait sur les plaies du Christ, comme un onguent salvateur, des touches d'aquarelle qui se diluaient dans l'eau. Fascinante intervention qui donnait au retable une nouvelle actualité.

Pour Yan Pei-Ming, récemment invité au Louvre, où il a exposé Les Funerailles de Monna Lisa, la réponse a été toute autre, à la mesure de sa démesure. Le pari était d'importance puisqu'il souhaitait se confronter à l'œuvre la plus illustre du monde, la Joconde. Il choisit une installation d'immenses toiles, développant à l'excès le paysage d'arrière-plan, représentant son père mort mais aussi lui-même. Lorsque je lui posais la question de ce choix pour un autoportrait peu modeste, il me répondit, par boutade : «Aucun artiste vivant ne peut entrer au Louvre ».

Thomas Huber. Selon moi, la question doit être envisagée dans une autre perspective, non pas en termes de juxtaposition entre art ancien et art contemporain, mais d'intégration du premier par le second. C'est l'attention que portent les artistes aux œuvres anciennes qui les rend actuelles, idée que je développe dans la peinture Kabinett der Bilder (2004; fig. 10). Réunissant en une seule image les œuvres exposées lors d'une rétrospective de mon œuvre tenue à la Aargauer Kunsthaus à Aarau et au Museum Boijmans van Beuningen à Rotterdam $(2004)^{9}$, elle exprime que le meilleur endroit pour conserver un tableau est dans les tableaux euxmêmes, et non pas dans un musée ou une galerie d'art. L'artiste devient ainsi garant de l'art ancien. Dans cette optique, Bogomir Ecker et moi-même avons donné le titre de "musée d'artistes » (Künstlermuseum) à notre intervention à Düsseldorf.

10. Thomas Huber, Kabinett der Bilder, 2004, Aarau, Aargauer Kunsthaus.
Notre intérêt ne portait ni sur la suite chronologique des œuvres, ni sur leur appartenance à une aire culturelle, ni surtout sur leur valeur marchande capitaliste, mais sur l'acte créatif qui a forgé les œuvres exposées. L'art actuel devrait en effet absorber les productions anciennes, les transformer et les actualiser. $C^{\prime}$ est ainsi en tout cas que des générations sont intervenues tout naturellement sur les héritages successifs. On a repeint des tableaux, utilisé des fragments de temples pour des linteaux ou des seuils de porte. On a recyclé l'art ancien,

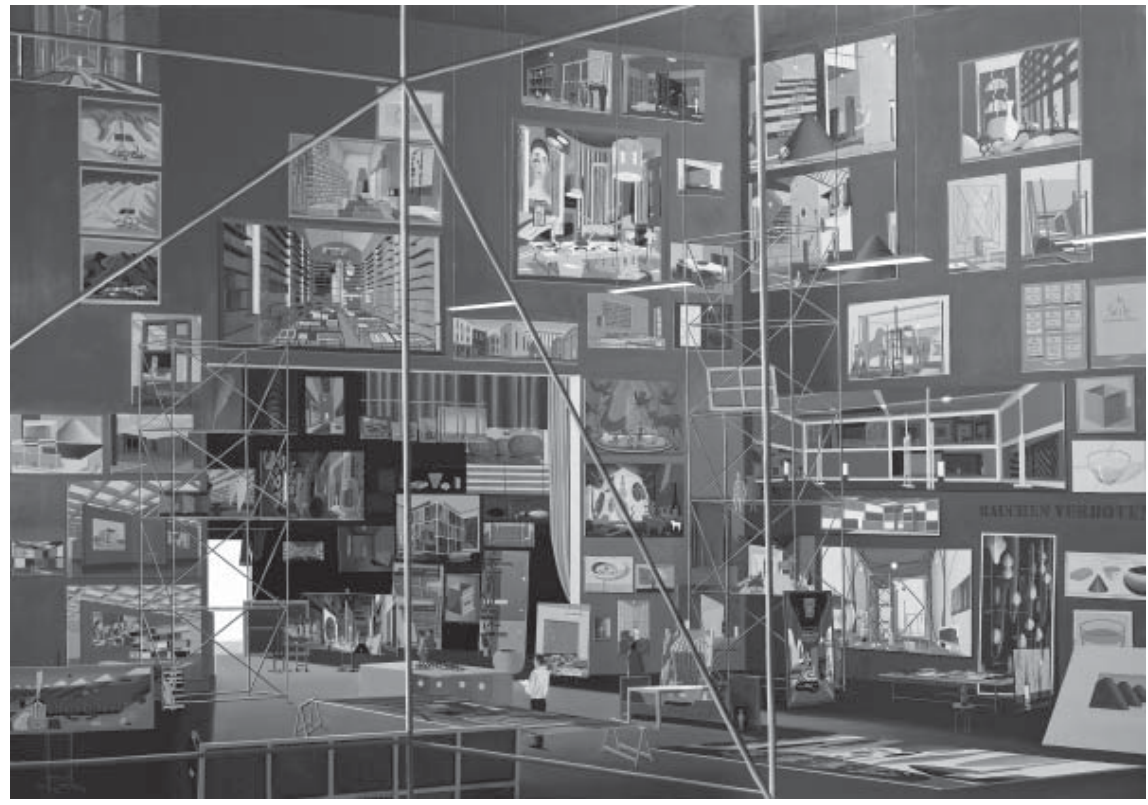


comme on le dirait aujourd'hui. Dans les réserves du musée de Düsseldorf, nous sommes tombés plusieurs fois sur des œuvres qui avaient été repeintes, modifiées ou complétées par les générations postérieures. Sur des tableaux, certaines parties ont été recouvertes par une nouvelle couche de peinture ou agrémentées de nouveaux personnages; des espaces encore vierges sur des feuilles à dessins ont servi à de nouvelles ébauches etc.

Faisant allusion à cette tradition, j'ai aussi annoncé que je me garderais le droit en tant qu'artiste d'actualiser certaines œuvres par des ajouts, de les améliorer ou de les repeindre simplement selon mes idées. On pourrait ainsi améliorer pas mal de choses grâce à de petites corrections ; combien d'erreurs pourrait-on faire disparaître par une nouvelle couche de peinture dans des œuvres d'art sinon bonnes et solides ? Un autre chapitre s'ouvre alors en vue des questions des droits de l'homme, des aspects politiques en fin de compte, de l'égalité des sexes, des égards portés sur des groupes sociaux marginaux, etc.

Car il n'y a pas qu'un seul point de vue sur les œuvres anciennes, qu'un seul canon autorisé. C'est le changement de perspective qui permet de développer le potentiel d'une collection. Évidemment, un commissaire d'exposition ou un conservateur de musée ne peut que se hérisser contre cette pratique. Sa position devra toutefois changer lorsqu'il ne disposera plus d'espace suffisant pour stocker les œuvres, ce qui risque d'arriver bientôt, les musées occidentaux emmagasinant trop d'œuvres et en acquérant en permanence de nouvelles. II fera alors sens d'actualiser les œuvres anciennes à l'aide de petites retouches, peut-être seulement minimales. À ce moment-là, la question posée ici deviendra superflue.

Laurent Salomé. J'ai eu l'occasion d'assister au cours des vingt dernières années à un retournement total des préjugés du monde politique : dans les années 1980, I'art contemporain gênait encore un peu, des projets radicaux pouvaient effrayer un maire et provoquer un flot de courriers injurieux. Ceci n'existe plus du tout aujourd'hui et c'est plutôt pour programmer une exposition sur le $X V I^{e}$ siècle qu'il faut se battre. Une forme d'art spectaculaire et ludique a définitivement rassuré et conquis les plus réticents et l'on remarque que c'est encore ce registre qui est privilégié dans les opérations de type "confrontation ». Il n'est pas exclu que, dans beaucoup de lieux, la pincée d'art contemporain ne soit devenue la potion indispensable pour dé-ringardiser un musée. II s'agirait alors d'un échec total et d'une pente catastrophique... Mais je me demande pourquoi j'utilise le conditionnel, nous y sommes! Aujourd'hui l'enjeu est de rendre I'art ancien vivant et vital sans avoir recours à des artifices. Faute de quoi on peut avoir les pires craintes sur l'avenir des musées. Les "inclusions» doivent être réservées aux travaux pour lesquels elles font sens et ce sens ne peut se trouver que dans le rapport personnel qu'entretient l'artiste concerné avec I'histoire de l'art ou dans I'inspiration profonde qu'il puise dans l'atmosphère du musée. À cet égard, la politique menée par le Musée Gassendi de Digne-les-Bains, largement appuyée sur ce type de face-à-face, avec des invitations d'artistes de tous les pays, est exemplaire. Autour de la figure de Pierre Gassendi, de l'histoire des sciences et plus précisément de celles du temps, dans une maison ancienne chargée d'atmosphère mais dotée d'une muséographie d'avant-garde, de grands artistes internationaux sont régulièrement invités à monter des expositions et à produire des œuvres. Le musée redevient un 
laboratoire, comme il l'était pour les artistes du XIXe siècle. Le travail est tellement mûri que certaines pièces, comme la collection de frottages de terres collectées dans la région par herman de vries (From Earth, 2001), présentés comme une sorte d'herbier, abolissent totalement le temps. L'activité du Musée Gassendi, où I'on peut voir aussi un cabinet Andy Goldsworthy et un cabinet Bernard Plossu, s'appuie également sur la Réserve géologique de HauteProvence, avec laquelle est cogéré le Centre d'Art Informel de Recherche sur la Nature (CAIRN). On est loin des effets purement décoratifs que nous infligent souvent les " confrontations», acier chromé sur boiserie dorée, high-tech sur vieille pierre, qui ne peuvent mener qu'à l'écœurement rapide.

Quel que soit le niveau de raffinement de la proposition, il reste une très large part du public qui ignore tout de la création actuelle et qui, fréquentant occasionnellement les musées, peut ressentir un déclic à la faveur d'une présence ponctuelle d'art contemporain, bien choisie et contenant une part suffisante de mystère et de poésie. Et tout événement de ce genre est très bon à prendre.

Nota bene : ce texte résulte de l'envoi de questions aux participants et d'un échange de courriels.

1. L'analyse que fait Bruno Latour de l'incapacité du concept de modernité à rendre compte de l'interpénétration contemporaine des domaines scientifique, social et culturel peut s'appliquer, selon nous, aux objets artistiques. Voir Bruno Latour, Nous n'avons jamais été modernes. Essai d'anthropologie symétrique, Paris, 1991.

2. François Morellet a réinvesti le Musée des beauxarts de Nantes en 2007 ; voir François Morellet. Ma musée, (cat. expo., Nantes, Musée des beaux-arts, 2007-2008), Lyon, 2007.

3. Jean-Hubert Martin en collaboration avec Dieter Scholz, "Musée des charmes », dans Künstlermuseum - Bogomir Ecker, Thomas Huber eine Neupräsentation der Sammlung des Museum Kunst Palast, Düsseldorf, 2002.

4. André Raffray ou la peinture recommencée, (cat. expo., Saint-Brieuc, Musée d'art et d'histoire/ Rouen, Musée des beaux-arts, 2005-2006), Paris, 2005.

5. Voir les expositions Picasso et les maîtres, (cat. expo., Paris, Galeries nationales du Grand Palais, 2008-2009), Paris, 2008, ou encore Ingres et les Modernes, (cat. expo., Québec, Musée national des beaux-arts/Montauban, Musée Ingres, 2009), Paris, 2008.

6. À travers le miroir, de Bonnard à Buren, (cat. expo., Rouen, Musée des beaux-arts, 2000-2001), Paris/Rouen, 2000.

7. Voir la n. 5.

8. Saint Clair Cemin a réalisé un certain nombre d'éléments décoratifs au sein du Musée de la Chasse et de la Nature : luminaires, garde-corps et main courante de l'escalier, piètements du mobilier muséographique. Conformément à la commande, son travail joue sur l'ambiguïté entre le végétal, I'animal et le minéral. Il constitue une sorte de leitmotiv qui unifie le parcours de visite.

9. Thomas Huber. Das Kabinett der Bilder, Beat Wismar éd., (cat. expo., Aarau, Aargauer Kunsthaus/ Rotterdam, Museum Boijmans Van Beuningen, 2004-2005), Aarau, 2004. 